\title{
Functional equation for partial zeta functions twisted by additive characters
}

by

\author{
Hugo Chapdelaine (Québec)
}

1. Introduction. Let $K$ be a number field of degree $n$ over $\mathbb{Q}$ and let $\left\{\sigma_{1}, \ldots, \sigma_{r_{1}}\right\}$ be a complete set of real embeddings of $K$ where $r_{1}+2 r_{2}=n$. Let $\omega$ be a sign character of $K$, i.e., a product over elements of a subset of the characters

$$
\operatorname{sign} \circ \sigma_{i}: K^{\times} \rightarrow \mathbb{R}^{\times} \rightarrow\{ \pm 1\} .
$$

Let $V$ be a lattice of maximal rank of $K$ and let

$$
\mathcal{O}_{V}=\{\alpha \in K: \alpha V \subseteq V\} .
$$

Note that $\mathcal{O}_{V}$ is an order of $K$ and $V$ is an invertible $\mathcal{O}_{V}$-ideal with inverse given explicitely by $V^{-1}=\left\{x \in K: x V \subset \mathcal{O}_{V}\right\}$. Let

$$
V^{*}=\left\{x \in K: \operatorname{Tr}_{K / \mathbb{Q}}(x v) \in \mathbb{Z} \text { for all } v \in V\right\}
$$

be the dual lattice of $V$. Note that $V^{*}$ is an invertible $\mathcal{O}_{V}$-module and that $V^{* *}=V$. For elements $a, b \in K$ we define

$$
\begin{aligned}
\Gamma_{a, b, V}=\left\{\varepsilon \in \mathcal{O}_{V}: \sigma_{i}(\varepsilon)>0 \forall i,\right. & (\varepsilon-1) a \in V, \\
& \left.(\varepsilon-1) b \in V^{*},(\varepsilon-1) a b \in \mathfrak{d}_{K}^{-1}\right\},
\end{aligned}
$$

where $\mathfrak{d}_{K}^{-1}=\left(\mathcal{O}_{K}\right)^{*}=\left\{x \in K: \operatorname{Tr}_{K / \mathbb{Q}}(x y) \in \mathbb{Z}\right.$ for all $\left.y \in \mathcal{O}_{K}\right\}$ is the inverse of the different ideal of $K$. One can verify that $\Gamma_{a, b, V}=\Gamma_{-b, a, V^{*}}$ is a subgroup of finite index in $\mathcal{O}_{K}^{\times}$.

For the set of data $(a, b, \omega, V)$ we define a partial zeta function twisted

2000 Mathematics Subject Classification: Primary 11R42.

Key words and phrases: zeta functions, number fields.

The author is grateful to the MPIM for the financial support during the writing of the paper. 
by an additive character as

$$
\Psi_{V}(a, b, \omega, s):=\left[\mathcal{O}_{K}: V\right]^{s} \sum_{\substack{v \in \mathfrak{R} \\ a+v \neq 0}} \omega(a+v) \frac{e^{2 \pi i \operatorname{Tr}_{K / \mathbb{Q}}(b(a+v))}}{\left|\mathbf{N}_{K / \mathbb{Q}}(a+v)\right|^{s}},
$$

where $\left[\mathcal{O}_{K}: V\right]$ is a positive rational number which plays the role of an index (see Definition 3.3), and $\mathfrak{R}=\left\{v_{i} \in V\right\}_{i \in I}$ is a complete set of representatives of $\{a+V\} / \Gamma_{a, b, V}$ in the sense that every element $0 \neq(a+v) \in a+V$ can be written uniquely as $\varepsilon\left(a+v_{i}\right)$ for some $v_{i} \in \mathfrak{R}$ and $\varepsilon \in \Gamma_{a, b, V}$. It is easy to see that (1.2) does not depend on the set of representatives $\mathfrak{R}$ and that it converges absolutely for any complex number $s$ such that $\operatorname{Re}(s)>1$.

Let $p=\left\{p_{i}\right\}_{i=1}^{r_{1}}$ be the signature of $\omega$, i.e., $\omega=\prod_{i=1}^{r_{1}}\left(\operatorname{sign} \circ \sigma_{i}\right)^{p_{i}}$ where $p_{i} \in\{0,1\}$. Then we define

$$
F_{V}^{p}(s / 2):=\left|d_{K}\right|^{s / 2} \pi^{-n s / 2} \prod_{i=1}^{r_{1}} \Gamma\left(\frac{s+p_{i}}{2}\right)\left(2^{1-s} \Gamma(s)\right)^{r_{2}},
$$

where $\mathbf{N}_{K / \mathbb{Q}}\left(\mathfrak{d}_{K}\right)=d_{K}$ is the discriminant of $K$ and $\Gamma(x)$ stands for the usual gamma function evaluated at $x$.

We can now state the main theorem which is proved in this paper.

THEOREM 1.1. Let

$$
Z_{V}(a, b, \omega, s)=F_{V}^{p}(s / 2) \Psi_{V}(a, b, \omega, s)
$$

be the completed zeta function of $\Psi_{V}\left(a, b, \omega_{p}, s\right)$. Then $Z_{V}(a, b, \omega, s)$ admits an analytic continuation to $\mathbb{C} \backslash\{0,1\}$ and has at most a pole of order one at $s \in\{0,1\}$. A pole of order one at $s=0$ occurs exactly when $p_{i}=0$ for all $i$ and $a \in V$. Similarly, a pole of order one at $s=1$ occurs exactly when $p_{i}=0$ for all $i$ and $-b \in V^{*}$. Moreover, $Z_{V}(a, b, \omega, s)$ satisfies the functional equation

$$
(-i)^{\operatorname{Tr}(p)} e^{-2 \pi i \operatorname{Tr}_{K / \mathbb{Q}}(a b)} Z_{V}(a, b, \omega, s)=Z_{V^{*}}(-b, a, \omega, 1-s) .
$$

The ideas which are used in the proof of Theorem 1.1 are due for the large part to Riemann and Hecke. Let

$$
\zeta_{\mathbb{Q}}(s)=\sum_{n \geq 1} \frac{1}{n^{s}}=\prod_{p} \frac{1}{1-1 / p^{s}}
$$

be the Riemann zeta function. The idea of using the transformation formula of the one-variable theta function $\theta(z)=\sum_{n \in \mathbb{Z}} e^{\pi i n^{2} z}$ to prove the functional equation

$$
\pi^{-s / 2} \Gamma(s / 2) \zeta_{\mathbb{Q}}(s)=\pi^{-(1-s) / 2} \Gamma((1-s) / 2) \zeta_{\mathbb{Q}}(1-s)
$$

is due to Riemann [Rie59]. Its generalisation to an arbitrary number field 
$K$ of degree $n$ over $\mathbb{Q}$, namely

$$
A^{s} \Gamma(s)^{r_{2}} \Gamma(s / 2)^{r_{1}} \zeta_{K}(s)=A^{1-s} \Gamma(1-s)^{r_{2}} \Gamma((1-s) / 2)^{r_{1}} \zeta_{K}(1-s),
$$

where

$$
\zeta_{K}(s)=\sum_{\mathfrak{a} \unlhd \mathcal{O}_{K}} \frac{1}{\mathbf{N}_{K / \mathbb{Q}}(\mathfrak{a})^{s}}=\prod_{\mathfrak{p}} \frac{1}{1-\frac{1}{\mathbf{N}_{K / \mathbb{Q}}(\mathfrak{p})^{s}}}
$$

and $A=2^{-r_{2}} \pi^{-n / 2} \sqrt{\left|d_{K}\right|}$, is due to Hecke [Hec17].

The functional equation (1.3) can be viewed, in some sense, as a natural generalisation of (1.4) and (1.5). However, there is one important aspect in which the zeta function $\Psi_{V}(a, b, \omega, s)$ differs from $\zeta_{K}(s)$, namely that in general, $\Psi_{V}(a, b, \omega, s)$ does not have any Euler product. The latter observation might be a reason why Hecke never published the functional equation (1.3).

It is clear that Hecke had at his disposal all the necessary tools to prove Theorem 1.1. In fact, in [Hec20], he proves a functional equation for the most general class of zeta functions which admit a degree one Euler product, namely for

$$
\zeta_{K}(\lambda, s)=\sum_{\mathfrak{a} \unlhd \mathcal{O}_{K}} \frac{\lambda(\mathfrak{a})}{\mathbf{N}_{K / \mathbb{Q}}(\mathfrak{a})^{s}}=\prod_{\mathfrak{p}} \frac{1}{1-\frac{\lambda(\mathfrak{p})}{\mathbf{N}_{K / \mathbb{Q}}(\mathfrak{p})^{s}}}
$$

where $\lambda$ is a so-called Größencharakter. It would be fair to say that the proof of the functional equation of $\zeta_{K}(\lambda, s)$ requires more ideas than the proof of Theorem 1.1. For example, Hecke introduced the notion of "idealer Zahlen" ( $\left.{ }^{1}\right)$ (see p. 17 (255) of [Hec20]) in order to work with complex numbers rather than ideals. These "idealer Zahlen" allowed him in particular to define certain Gauss sums (depending on $\lambda$ ) which play a crucial role in the proof of the functional equation of $\zeta_{K}(\lambda, s)$.

The zeta function $\Psi_{V}\left(a, b, \omega_{p}, s\right)$ arose naturally in some of the previous work of the author (see for example [Cha07a]). Let us explain in more detail the context in which it arose. Let $K$ be a totally real number field and let $V=\mathfrak{a} / \mathfrak{f} \mathfrak{d}_{K}$ where $\mathfrak{a}, \mathfrak{f}$ are integral ideals which are coprime. Assume that the sign character $\omega_{p}$ is chosen so that $\omega_{p}=1$ or $\omega_{p}=\operatorname{sign} \circ \mathbf{N}_{K / \mathbb{Q}}$. In [Cha07b], it is explained how the special values at negative integers of

$$
\Psi_{V^{*}}\left(1,0, \omega_{p}, s\right)=\mathbf{N}_{K / \mathbb{Q}}(\mathfrak{f})^{s}\left(\sum_{\left\{0 \neq \mu \in 1+\mathfrak{f} \mathfrak{a}^{-1}\right\} / \Gamma_{\mathfrak{a}}} \frac{\omega_{p}(\mu)}{\left|\mathbf{N}_{K / \mathbb{Q}}(\mu \mathfrak{a})\right|^{s}}\right)
$$

(see equation (5.1) for more details) can be related to special values at

$\left({ }^{1}\right)$ The English translation of idealer Zahlen is ideal numbers. These ideal numbers, which have the drawback of not being defined in a canonical way, can be viewed in some sense as a precursor to the notion of idèles introduced by Chevalley in the mid-1930s. 
negative integers of classical partial zeta functions, namely

$$
\begin{aligned}
\zeta(\mathfrak{a}, \mathfrak{f} \infty, s) & :=\mathbf{N}_{K / \mathbb{Q}}(\mathfrak{a})^{-s} \sum_{\Gamma_{\mathfrak{a}} \backslash\left\{\lambda \in \mathfrak{a}^{-1}: \lambda \equiv 1\left(\bmod \mathfrak{f a} \mathfrak{a}^{-1}\right), \lambda \gg 0\right\}} \frac{1}{\left|\mathbf{N}_{K / \mathbb{Q}}(\lambda)\right|^{s}} \\
& =\sum_{\substack{\mathfrak{b} \subseteq \mathcal{O}_{K} \\
\mathfrak{b} \sim \mathfrak{a} \mathfrak{a}}} \frac{1}{\mathbf{N}_{K / \mathbb{Q}}(\mathfrak{b})^{s}},
\end{aligned}
$$

where $\mathfrak{b} \sim_{\mathfrak{f}} \mathfrak{a}$ means that $\mathfrak{b}$ and $\mathfrak{a}$ lie in the same narrow ray ideal class modulo $\mathfrak{f}$, and $\Gamma_{\mathfrak{a}}=\mathcal{O}_{K}(\infty)^{\times} \cap\left(1+\mathfrak{f a}^{-1}\right)$ where $\mathcal{O}_{K}(\infty)^{\times}$denotes the group of totally positive units of $\mathcal{O}_{K}$. Note that the summation in (1.8) is taken over totally positive elements of $\mathfrak{a}^{-1}$. Special values of $\zeta(\mathfrak{a}, \mathfrak{f} \infty, s)$ at negative integers turns out to be rational numbers (see [Kli62], [Sie69] and [Shi76]). These rational numbers satisfy many remarkable congruence relations which have been exploited by many number theorists to construct various $p$-adic objects. In particular, one can construct $p$-adic $L$-functions which interpolate these special values (see [DR80] and [CN79]). In the last section of this paper we show essentially that unless $K$ is a totally real number field and $\omega_{p}=1$ or sign $\circ \mathbf{N}_{K / \mathbb{Q}}$, no such $p$-adic $L$-function exists since the special values at negative integers of (1.7) are all equal to zero.

When the author wrote the paper, he was unaware that a special case of the functional equation (1.3) had already appeared in a paper of Siegel (see equation (10) of [Sie70]). In the paragraph below that equation, Siegel writes:

Es ist sonderbar, daß (10) bisher in der Literatur nicht erwähnt worden ist. Auch wenn man die Funktionalgleichung der $L$-Reihen im Auge hat, erscheint es übrigens durchsichtiger, zunächst die einfachere Formel (10) zu beweisen und erst nachher die notwendigen algebraischen arithmetischen Sätze über Charaktere herzuleiten.

Thirty eight years later, the author of this paper shares the exact same view.

Finally, let us mention some connection between the Lerch zeta function and the zeta function $\Psi_{V}(a, b, \omega, s)$ in the case where $K=\mathbb{Q}$. For real numbers $0<u, v \leq 1$ consider the Lerch zeta function

$$
\varphi(u, v, s)=\sum_{n=0}^{\infty} \frac{e^{2 \pi i n v}}{|n+u|^{s}}, \quad \operatorname{Re}(s)>1 .
$$

Let $\omega=1, V=\mathbb{Z}$ and $a, b \in \mathbb{Q} \cap(0,1)$. Then a direct computation shows that

$$
\Psi_{V}(a, b, \omega, s)=e^{2 \pi i a b} \sum_{n \in \mathbb{Z}} \frac{e^{2 \pi i b n}}{|n+a|^{s}}=e^{2 \pi i a b}(\varphi(a, b, s)+\varphi(1-a, b, s)) .
$$


The contribution of this paper consists essentially in filling a gap in the literature by providing a detailed proof of the functional equation (1.3). In writting the paper, we have decided to follow a more modern account of the work of Riemann and Hecke on zeta functions: namely we borrow most of our notation from Chapter 7 of Neukirch's book [Neu99] on algebraic number theory. The interested reader may consult as well that chapter in order to find a proof of the functional equation for $\zeta_{K}(\lambda, s)$.

2. Notation. Let $K$ be a number field of degree $n$ over $\mathbb{Q}$ and let $X=\operatorname{Hom}(K, \mathbb{C})$ be a complete set of embeddings of $K$ into $\mathbb{C}$. The set $X$ can be written in the following way:

$$
X=\left\{\sigma_{1}, \ldots, \sigma_{r_{1}}, \varrho_{1}, \bar{\varrho}_{1}, \ldots, \varrho_{r_{2}}, \bar{\varrho}_{r_{2}}\right\},
$$

where $r_{1}+2 r_{2}=n$, the $\sigma_{i}$ 's are the real embeddings, the $\varrho_{i}$ 's are the complex embeddings such that $c \circ \varrho_{i}=\bar{\varrho}_{i}$ where $c: \mathbb{C} \rightarrow \mathbb{C}$ corresponds to complex conjugation. Note that up to permutation there is still a choice in our way of writing the set $X$ which corresponds to a choice of a privileged representative $\varrho_{i}$ for every pair of complex embeddings of $K$. Usually, for an element $a \in \mathbb{C}$ we will denote its complex conjugate $c(a)$ by $\bar{a}$. Note that $X$ is naturally a left $\operatorname{Gal}(\mathbb{C} / \mathbb{R})$-set. For $\tau \in X$ we will also denote $c \circ \tau$ by $\bar{\tau}$.

We consider the $n$-dimensional $\mathbb{C}$-algbera attached to $X$,

$$
\mathbf{C}_{X}:=\prod_{\tau \in X} \mathbb{C}
$$

of all tuples $z=\left(z_{\tau}\right)_{\tau \in X}, z_{\tau} \in \mathbb{C}$, with componentwise addition and multiplication. Since the subset $X$ is fixed from the beginning we will denote $\mathbf{C}_{X}$ simply by $\mathbf{C}$.

For the remainder of the paper we will use the set of notations attached to $\mathbf{C}$ which is introduced on pages 444 and 445 of [Neu99]. The $\mathbb{C}$-algebra $\mathbf{C}$ is endowed with three involutions. For every element $z=\left(z_{\tau}\right)_{\tau \in X} \in \mathbf{C}$ we define the elements $z^{*},{ }^{*} z, \bar{z} \in \mathbf{C}$ as

$$
\left(z^{*}\right)_{\tau}=z_{\bar{\tau}}, \quad\left({ }^{*} z\right)_{\tau}=\overline{\left(z_{\tau}\right)}, \quad(\bar{z})_{\tau}=\left({ }^{*} z^{*}\right)_{\tau}=\overline{\left(z_{\bar{\tau}}\right)} .
$$

The $\mathbb{C}$-algebra $\mathbf{C}$ is equipped with certain distinguished subsets, namely

(1) $\mathbf{R}=\{z \in \mathbf{C}: \bar{z}=z\}$,

(2) $\mathbf{R}_{ \pm}=\left\{x \in \mathbf{R}: x=x^{*}\right\}$,

(3) $\mathbf{R}_{+}^{\times}=\left\{x \in \mathbf{R}_{ \pm}: x>0\right\}$,

(4) $\mathbf{H}=\mathbf{R}_{ \pm}+i \mathbf{R}_{+}^{\times}$.

If $\delta \in \mathbb{R}$, the notation $x>\delta$ means that $x_{\tau}>\delta$ for all $\tau \in X$. By definition we have the following inclusions:

$$
\mathbf{H} \subseteq \mathbf{C} \supseteq \mathbf{R} \supseteq \mathbf{R}_{ \pm} \supseteq \mathbf{R}_{+}^{\times} .
$$

Note that the subset $\mathbf{R}$ is naturally an $\mathbb{R}$-subalgebra of the $\mathbb{C}$-algebra $\mathbf{C}$. 
For every infinite place $\nu$ of $K$ there exists a unique field inclusion $\iota_{\nu}: \mathbb{R} \rightarrow K_{\nu}$. Because of the uniqueness of $\iota_{\nu}$ we can view the set $\mathbb{R}$ as being naturally included in $K_{\nu}$. We thus have a natural isomorphism $K \otimes_{\mathbb{Q}} \mathbb{R} \rightarrow \prod_{\nu \mid \infty} K_{\nu}$ given by $\alpha \otimes \beta \mapsto(\alpha \beta)_{\nu \mid \infty}$. Our choice of a complete set of pairwise non-conjugate complex embeddings $\left\{\varrho_{i}\right\}_{i=1}^{r_{2}}$ gives rise to a natural isomorphism $\prod_{\nu \mid \infty} K_{\nu} \rightarrow \mathbf{R}$ given by $\left(x_{\nu}\right)_{\nu \mid \infty} \mapsto\left(y_{\tau}\right)_{\tau \in X}$ where $y_{\tau}=x_{\nu}$ if $\tau$ is the real embedding corresponding to the real place $\nu$, and $y_{\tau}=x_{\nu}\left(\right.$ resp. $\left.y_{\tau}=\bar{x}_{\nu}\right)$ if $\tau=\varrho_{i}$ (resp. $\tau=\bar{\varrho}_{i}$ ) is a complex embedding corresponding to the complex place $\nu$. In this way we obtain a natural isomorphism of $\mathbb{R}$-algebras

$$
\iota: K \otimes_{\mathbb{Q}} \mathbb{R} \stackrel{\sim}{\rightarrow} \mathbf{R} .
$$

From now on we will think of the number field $K$ as being naturally included in the $\mathbb{R}$-algebra $K \otimes_{\mathbb{Q}} \mathbb{R}$ via the natural map $\alpha \mapsto \alpha \otimes 1$.

The $\mathbb{C}$-algebra $\mathbf{C}$ and certain of its subsets are equipped with various maps. For the additive group $\mathbf{C}$ (resp. multiplicative group $\mathbf{C}^{\times}$) we have the homomorphisms

$$
\operatorname{Tr}: \mathbf{C} \rightarrow \mathbb{C}, \quad \operatorname{Tr}(z)=\sum_{\tau} z_{\tau}, \quad N: \mathbf{C}^{\times} \rightarrow \mathbb{C}^{\times}, \quad N(z)=\prod_{\tau} z_{\tau} .
$$

We have on $\mathbf{C}$ an hermitian scalar product

$$
\langle x, y\rangle=\sum_{\tau} x_{\tau} \bar{y}_{\tau}=\operatorname{Tr}\left(x\left(^{*} y\right)\right) \quad \text { and } \quad\|z\|=\sqrt{\langle z, z\rangle} .
$$

It is invariant under conjugation, i.e., $\overline{\langle x, y\rangle}=\langle\bar{x}, \bar{y}\rangle$, and restricting it yields a euclidian metric on the $\mathbb{R}$-vector space $\mathbf{R}$. If $z \in \mathbf{C}$, then ${ }^{*} z$ is the adjoint element, i.e., $\langle x z, y\rangle=\left\langle x,{ }^{*} z y\right\rangle$. For two tuples $z=\left(z_{\tau}\right)_{\tau},\left(p_{\tau}\right)_{\tau} \in \mathbf{C}$ the power

$$
z^{p}=\left(z_{\tau}^{p_{\tau}}\right) \in \mathbf{C} \quad \text { where } \quad z_{\tau}^{p_{\tau}}=e^{p_{\tau} \log z_{\tau}},
$$

is well defined if we agree to take the principal branch of logarithm and assume that the $z_{\tau}$ 's move only in the plane cut along the negative real axis. Finally we define

$$
\begin{aligned}
&\|\|: \mathbf{R}^{\times} \rightarrow \mathbf{R}_{+}^{\times}, x=\left(x_{\tau}\right)_{\tau} \mapsto\|x\|=\left(\left|x_{\tau}\right|\right)_{\tau}, \\
& \log : \mathbf{R}_{+}^{\times} \stackrel{\sim}{\rightarrow} \mathbf{R}_{ \pm}, \quad x=\left(x_{\tau}\right)_{\tau} \mapsto \log x=\left(\log x_{\tau}\right)_{\tau} .
\end{aligned}
$$

\section{Multivariable $\theta$-function and $\Gamma$-function}

Definition 3.1. We say that a tuple $p=\left(p_{\tau}\right)_{\tau \in X}$ of non-negative integers is admissible (resp. strictly admissible) if $p_{\tau} \in\{0,1\}$ when $\bar{\tau}=\tau$ and $p_{\tau} p_{\bar{\tau}}=0$ (resp. $\left.p_{\tau}=p_{\bar{\tau}}=0\right)$ if $\tau \neq \bar{\tau}$. 
Definition 3.2. Let $V \subseteq \mathbf{R}$ be a lattice of maximal rank, let $a, b \in \mathbf{R}$ and let $p \in \prod_{\tau \in X} \mathbb{Z}$ be admissible. We define the theta series

$$
\theta_{V}^{p}(a, b, z)=\sum_{v \in V} N\left((a+v)^{p}\right) e^{\pi i\langle(a+v) z,(a+v)\rangle} e^{2 \pi i\langle b, a+v\rangle},
$$

which converges absolutely for every $z \in \mathbf{H}$.

REMARK 3.1. Note that our definition of $\theta_{V}^{p}(a, b, z)$ is slightly different from the one appearing on the bottom of page 450 of [Neu99].

DeFinition 3.3. Let $V$ be a lattice of maximal rank in $K$. Let $\left\{e_{1}, \ldots, e_{n}\right\}$ be a $\mathbb{Z}$-basis of $\mathcal{O}_{K}$ and let $\left\{e_{1}^{\prime}, \ldots, e_{n}^{\prime}\right\}$ be a $\mathbb{Z}$-basis of $V$. Let $M \in M_{n}(\mathbb{Q})$ be the matrix which sends the ordered basis $\left(e_{i}\right)_{i=1}^{n}$ to the ordered basis $\left(e_{i}^{\prime}\right)_{i=1}^{n}$. Then we define the index $\left[\mathcal{O}_{K}: V\right]$ to be the rational number $|\operatorname{det}(M)|$.

It is easy to see that $\left[\mathcal{O}_{K}: V\right]$ is a well defined positive rational number independent of the choice of the bases. It is also convenient to define the positive rational number

$$
d_{V}=\left[\mathcal{O}_{K}: V\right]^{2}\left|d_{K}\right|,
$$

where $d_{K}$ is the discriminant of $K$. The quantity $\sqrt{d_{V}}$ can be interpreted as the covolume of the lattice $\iota(V) \subset \mathbf{R}$ with respect to the Haar measure $d x$ on $(\mathbf{R},\langle\rangle$,$) which ascribes the volume 1$ to the cube spanned by an orthonormal basis.

The key ingredient to prove the functional equation appearing in Theorem 1.1 is the following transformation formula for the theta function.

Theorem 3.1 (theta transformation formula). Let $a, b \in \mathbf{R}$ and let $p \in$ $\prod_{\tau} \mathbb{Z}$ be admissible. Then

$$
\begin{array}{r}
\theta_{V}^{p}(a, b,-1 / z)=\left(i^{\operatorname{Tr}(p)} e^{-2 \pi i\langle a, b\rangle} \sqrt{d_{V}}\right)^{-1} N\left((z / i)^{p+\frac{1}{2} \cdot \mathbf{1}}\right) \theta_{V^{*}}^{p}(-b, a, z) \\
\text { for all } z \in \mathbf{H},
\end{array}
$$

where $\mathbf{1}$ is the unit element in $\mathbf{R}$ and $V^{*}$ is the dual lattice of $V$, i.e.,

$$
V^{*}=\left\{v^{\prime} \in \mathbf{R}:\left\langle v^{\prime}, v\right\rangle \in \mathbb{Z} \text { for all } v \in V\right\} .
$$

Proof. See equation (19) on p. 26 (264) of [Hec20] or (3.6) on page 454 of [Neu99].

We have

$$
\mathbf{R}_{+}^{\times}=\prod_{\nu \mid \infty} \mathbf{R}_{+, \nu}^{\times} \quad \text { and } \quad \mathbf{R}_{ \pm}^{\times}=\prod_{\nu \mid \infty} \mathbf{R}_{ \pm, \nu}^{\times}
$$

where $\mathbf{R}_{+, \nu}^{\times}=\mathbb{R}_{+}^{\times}$(resp. $\mathbf{R}_{ \pm, \nu}^{\times}=\mathbb{R}_{ \pm}^{\times}$) if $\nu$ is real and $\mathbf{R}_{+, \nu}^{\times}=\{(y, y)$ : $\left.y \in \mathbb{R}_{+}^{\times}\right\}$(resp. $\mathbf{R}_{ \pm, \nu}^{\times}=\left\{(y, y): y \in \mathbb{R}_{ \pm}^{\times}\right\}$) if $\nu$ is complex. We define isomorphisms $\mathbf{R}_{+, \nu}^{\times} \rightarrow \mathbb{R}_{+}^{\times}$given by $y \mapsto y$ if $\nu$ is real and $(y, y) \mapsto y^{2}$ if $\nu$ is complex. We thus obtain an isomorphism $\mathbf{R}_{+}^{\times} \rightarrow \prod_{\nu \mid \infty} \mathbb{R}_{+}^{\times}$. We denote 
by $\frac{d y}{y}$ the Haar measure on $\mathbf{R}_{+}^{\times}$which corresponds to the pull back of the product measure $\prod_{\nu} \frac{d t}{t}$ where $\frac{d t}{t}$ is the usual Haar measure on $\mathbb{R}_{+}^{\times}$. The Haar measure thus defined is called the canonical measure on $\mathbf{R}_{+}^{\times}$. Consider the isomorphism

$$
\mathbf{R}_{+}^{\times} \stackrel{\log }{\longrightarrow} \mathbf{R}_{ \pm} \stackrel{j}{\rightarrow} \prod_{\nu \mid \infty} \mathbb{R}
$$

where $j: \mathbf{R}_{ \pm, \nu} \rightarrow \mathbb{R}$ is given by $x_{\nu} \mapsto x_{\nu}$ (resp. $\left.\left(x_{\nu}, x_{\nu}\right) \mapsto 2 x_{\nu}\right)$ if $\nu$ is real (resp. if $\nu$ is complex). Then the canonical Haar measure $\frac{d y}{y}$ pushes forward to the Lebesgue measure on $\prod_{\nu \mid \infty} \mathbb{R}$.

Definition 3.4. For $\mathbf{s}=\left(s_{\tau}\right)_{\tau} \in \mathbf{C}$ such that $\operatorname{Re}\left(s_{\tau}\right)>0$ and $p=$ $\left(p_{\tau}\right)_{\tau}$ an admissible tuple, we define the gamma function associated to the $\operatorname{Gal}(\mathbb{C} / \mathbb{R})$-set $X$ as

$$
\Gamma_{X}^{p}(\mathbf{s})=\int_{\mathbf{R}_{+}^{\times}} N\left(e^{-y} y^{\mathbf{s}+\frac{1}{2} p}\right) \frac{d y}{y},
$$

where $y=\left(y_{\tau}\right)_{\tau} \in \mathbf{R}_{+}^{\times}, e^{-y}=\left(e^{-y_{\tau}}\right)_{\tau}$ and $y^{\mathbf{s}+\frac{1}{2} p}=\left(e^{\left(s_{\tau}+\frac{1}{2} p_{\tau}\right) \log y_{\tau}}\right)_{\tau}$.

Using (3.1) we can write $\Gamma_{X}^{p}(\mathbf{s})$ as

$$
\Gamma_{X}^{p}(\mathbf{s})=\prod_{\nu \mid \infty} \Gamma_{\nu}^{p_{\nu}}\left(\mathbf{s}_{\nu}\right)
$$

where $\mathbf{s}_{\nu}=s_{\sigma_{i}}$ (resp. $p_{\nu}=p_{\sigma_{i}}$ ) if $\nu$ is the real place corresponding to $\sigma_{i}$ and $\mathbf{s}_{\nu}=\left(s_{\varrho_{i}}, s_{\bar{\varrho}_{i}}\right)$ (resp. $\left.p_{\nu}=\left(p_{\varrho_{i}}, p_{\bar{\varrho}_{i}}\right)\right)$ if $\nu$ is the complex place corresponding to $\varrho_{i}$. The factors are given explicitly by

$$
\Gamma_{\nu}^{p_{\nu}}\left(\mathbf{s}_{\nu}\right)= \begin{cases}\Gamma\left(\mathbf{s}_{\nu}+\frac{1}{2} p_{\nu}\right) & \text { if } \nu \text { is real, } \\ 2^{1-\operatorname{Tr}\left(\mathbf{s}_{\nu}+\frac{1}{2} p_{\nu}\right)} \Gamma\left(\operatorname{Tr}\left(\mathbf{s}_{\nu}+\frac{1}{2} p_{\nu}\right)\right) & \text { if } \nu \text { is complex }\end{cases}
$$

where $\Gamma(x)$ is the usual one-variable gamma function.

4. Proof of the functional equation. Consider the multivariable gamma function

$$
\Gamma_{X}^{p}(s)=\int_{\mathbf{R}_{+}^{\times}} N\left(e^{-y} y^{s \mathbf{1}+\frac{1}{2} p}\right) \frac{d y}{y},
$$

where $s \in \mathbb{C}, \operatorname{Re}(s)>0, \mathbf{1}$ is the unit of $\mathbf{C}$ and $p=\left(p_{\tau}\right)_{\tau}$ is an admissible tuple. Let $V$ be a lattice of maximal rank in $K$ and let $a, b \in \mathbf{R}$. In the integral of (4.1) we substitute

$$
y \mapsto \pi|a+v|^{2} y / d_{V}^{1 / n}
$$


where || denotes the map $\mathbf{R}^{\times} \rightarrow \mathbf{R}_{+}^{\times},\left(x_{\tau}\right)_{\tau} \mapsto\left(\left|x_{\tau}\right|\right)_{\tau}$. We then obtain

$$
\begin{aligned}
& \int_{\mathbf{R}_{+}^{\times}} e^{-\pi\left\langle(a+v) y / d_{V}^{1 / n},(a+v)\right\rangle} N\left(y^{s \mathbf{1}+\frac{1}{2} p}\right) \frac{d y}{y} \\
= & \pi^{-\operatorname{Tr}\left(\frac{1}{2} p\right)}\left(\left|d_{V}\right|^{1 / n}\right)^{\operatorname{Tr}\left(\frac{1}{2} p\right)}\left|d_{K}\right|^{s} \pi^{-n s} \Gamma_{X}^{p}(s) \frac{\left[\mathcal{O}_{K}: V\right]^{2 s}}{\left|N\left((a+v)^{p}\right)\right||N(a+v)|^{2 s}}
\end{aligned}
$$

for $\operatorname{Re}(s)>1$.

Remember that $\mathcal{O}_{V}=\{\alpha \in K: \alpha V \subseteq V\}$. Denote by $\mathfrak{d}_{K}$ the discriminant ideal of $K$, i.e., $\mathfrak{d}_{K}^{-1}=\left\{x \in K: \operatorname{Tr}_{K / \mathbb{Q}}(x y) \in \mathbb{Z}\right.$ for all $\left.y \in \mathcal{O}_{K}\right\}$. From now on we identify $V$ with $\iota(V) \subseteq \mathbf{R}$. We define

$$
\begin{aligned}
\Gamma_{a, b, V}=\left\{\varepsilon \in \mathcal{O}_{V}: \sigma_{i}(\varepsilon)>\right. & 0 \forall i,(\varepsilon-1) a \in \iota(V), \\
& \left.(\varepsilon-1) b \in \iota\left(V^{*}\right),(\varepsilon-1) a b \in \iota\left(\mathfrak{d}_{K}^{-1}\right)\right\} .
\end{aligned}
$$

One can verify that the subgroup $\Gamma_{a, b, V}$ has finite index in $\mathcal{O}_{K}^{\times}$. Let $\varepsilon \in \Gamma_{a, b, V}$ and let $v \in V$ be such that $a+v \neq 0$. Since $\varepsilon \in \Gamma_{a, b, V}$ we have $\varepsilon(a+v)=a+v^{\prime}$ for (a unique) $v^{\prime} \in V$. Assume furthermore that the tuple $p=\left(p_{\tau}\right)_{\tau}$ is strictly admissible. Then a direct computation shows that

$$
\frac{N\left((a+v)^{p}\right)}{\left|N\left((a+v)^{p}\right)\right|} \frac{e^{2 \pi i\langle b, a+v\rangle}}{|N(a+v)|^{2 s}}=\frac{N\left(\left(a+v^{\prime}\right)^{p}\right)}{\left|N\left(\left(a+v^{\prime}\right)^{p}\right)\right|} \frac{e^{2 \pi i\left\langle b, a+v^{\prime}\right\rangle}}{\left|N\left(a+v^{\prime}\right)\right|^{2 s}} .
$$

Note that $x \mapsto \mathbf{N}\left(x^{p}\right) /\left|\mathbf{N}\left(x^{p}\right)\right|$ is nothing else than a sign character of $K$, i.e., a group homomorphism $\omega_{p}:\left(K \otimes_{\mathbb{Q}} \mathbb{R}\right)^{\times} \rightarrow\{ \pm 1\}$.

Recall that $\mathfrak{R}=\left\{v_{i} \in V\right\}_{i \in I}$ is a complete set of representatives of $\{a+V\} / \Gamma_{a, b, V}$ in the sense that every element $0 \neq a+v \in a+V$ can be written uniquely as $\varepsilon\left(a+v_{i}\right)$ for some $v_{i} \in \mathfrak{R}$ and $\varepsilon \in \Gamma_{a, b, V}$. Let $\omega_{p}$ be the sign character associated to the strictly admissible tuple $p=\left(p_{\tau}\right)_{\tau}$. Using (4.2) and (4.4) we deduce that

$$
\begin{aligned}
\frac{1}{C} \int_{\mathbf{R}_{+}^{\times}} & \left(\widetilde{\theta}_{V}^{p}\left(a, b, i y / d_{V}^{1 / n}\right)-c_{V}^{p}(a, b)\right) N\left(y^{s+\frac{1}{2} p}\right) \frac{d y}{y} \\
& =\left|d_{K}\right|^{s} \pi^{-n s}\left[\mathcal{O}_{K}: V\right]^{2 s} \Gamma_{X}^{p}(s) \sum_{\substack{v \in \mathfrak{R} \\
a+v \neq 0}} \omega_{p}(a+v) \frac{e^{2 \pi i\langle b, a+v\rangle}}{|N(a+v)|^{2 s}} \\
& =\left|d_{K}\right|^{s} \pi^{-n s} \Gamma_{X}^{p}(s) \Psi_{V}\left(a, b, \omega_{p}, 2 s\right),
\end{aligned}
$$

where

$$
\widetilde{\theta}_{V}^{p}\left(a, b, z / d_{V}^{1 / n}\right)=\sum_{\substack{v \in \mathfrak{R} \\ a+v \neq 0}} N\left((a+v)^{p}\right) e^{\pi i\left\langle(a+v) z / d_{V}^{1 / n},(a+v)\right\rangle} e^{2 \pi i\langle b, a+v\rangle}
$$


for $z \in \mathbf{H}$, and

$$
c_{V}^{p}(a, b)=\lim _{z \rightarrow i \infty} \widetilde{\theta}_{V}^{p}\left(a, b, z / d_{V}^{1 / n}\right) \quad \text { and } \quad C=\pi^{-\operatorname{Tr}\left(\frac{1}{2} p\right)}\left(\left|d_{V}\right|^{1 / n}\right)^{\operatorname{Tr}\left(\frac{1}{2} p\right)} .
$$

Note that $c_{V}^{p}(a, b)=0$ unless $p_{\tau}=0$ for all $\tau \in X$ and $a \in V$. In the latter case we have $c_{V}^{p}(a, b)=1$.

Definition 4.1. We define the completed zeta function $Z_{V}\left(a, b, \omega_{p}, 2 s\right)$ to be

$$
Z_{V}\left(a, b, \omega_{p}, 2 s\right):=F_{V}^{p}(s) \Psi\left(a, b, \omega_{p}, 2 s\right),
$$

where $F_{V}^{p}(s)=\left|d_{K}\right|^{s} \pi^{-n s} \Gamma_{X}^{p}(s)$.

The image of $\Gamma_{a, b, V}$ under the mapping ||$: \mathbf{R}^{\times} \rightarrow \mathbf{R}_{+}^{\times}$is contained in the norm-one hypersurface

$$
\mathbf{S}=\left\{x \in \mathbf{R}_{+}^{\times}: N(x)=1\right\} .
$$

We can write every $y \in \mathbf{R}_{+}^{\times}$in the form

$$
y=x t^{1 / n}, \quad x=\frac{y}{N(y)^{1 / n}}, \quad t=N(y) .
$$

We thus obtain a direct decomposition

$$
\mathbf{R}_{+}^{\times}=\mathbf{S} \times \mathbb{R}_{+}^{\times} .
$$

We let $d^{*} x$ be the unique Haar measure on the multiplicative group $\mathbf{S}$ such that the canonical Haar measure $\frac{d y}{y}$ on $\mathbf{R}_{+}^{\times}$becomes the product measure

$$
\frac{d y}{y}=d^{*} x \times \frac{d t}{t} .
$$

Proposition 4.1. The completed zeta function $Z_{V}\left(a, b, \omega_{p}, 2 s\right)$ is the Mellin transform

$$
Z_{V}\left(a, b, \omega_{p}, 2 s\right)=L(f, s)=\int_{0}^{\infty}(f(t)-f(\infty)) t^{s} \frac{d t}{t}, \quad \operatorname{Re}(s)>1,
$$

of the function

$$
f(t)=\frac{1}{C} \int_{\mathfrak{F}} \theta_{V}^{p}\left(a, b, \omega_{p}, i x t^{1 / n} / d_{V}^{1 / n}\right) N\left(\left(i x t^{1 / n} / d_{V}^{1 / n}\right)^{\frac{1}{2} p}\right) d^{*} x
$$

where $\mathfrak{F}$ is a fundamental domain for the action of $\iota\left(\Gamma_{a, b, V}\right) \subseteq \mathbf{S}$ on $\mathbf{S}$,

$$
C=\pi^{-\operatorname{Tr}\left(\frac{1}{2} p\right)}\left(\left|d_{V}\right|^{1 / n}\right)^{\operatorname{Tr}\left(\frac{1}{2} p\right)} \quad \text { and } \quad f(\infty)=\frac{c_{V}^{p}(a, b)}{C} \operatorname{vol}(\mathfrak{F}) .
$$

Proof. This is the same argument as the proof of Proposition (5.5) of [Neu99]. 
Lemma 4.1. The fundamental domain $\mathfrak{F}$ of $\mathbf{S}$ has the following volume with respect to the measure $d^{*} x$ :

$$
\operatorname{vol}(\mathfrak{F})=\left[\mathcal{O}_{K}^{\times}: \Gamma_{a, b, V}\right] R_{K},
$$

where $R_{K}$ is the regulator of $K$.

Proof. This is the same argument as Lemma (5.6) of [Neu99].

Proposition 4.2. The function

$$
t \mapsto f(t)=\frac{1}{C} \int_{\mathfrak{F}} \theta_{V}^{p}\left(a, b, \omega_{p}, i x t^{1 / n} / d_{V}^{1 / n}\right) N\left(\left(i x t^{1 / n} / d_{V}^{1 / n}\right)^{\frac{1}{2} p}\right) d^{*} x
$$

for $t \in \mathbb{R}_{+}$satisfies the functional equation

$$
f(1 / t)=\left(i^{\operatorname{Tr}(p)} e^{-2 \pi i\langle a, b\rangle}\right)^{-1} \sqrt{t} g(t),
$$

where

$$
g(t)=\frac{1}{C} \int_{\mathfrak{F}^{\prime}} \theta_{V^{*}}^{p}\left(-b, a, \omega_{p}, i x t^{1 / n} / d_{V^{*}}^{1 / n}\right) N\left(\left(i x t^{1 / n} / d_{V}^{1 / n}\right)^{\frac{1}{2} p}\right) d^{*} x,
$$

and $\mathfrak{F}^{\prime}$ is a fundamental domain for the action of $\iota\left(\Gamma_{-b, a, V^{*}}\right)$ on $\mathbf{S}$ and $\Gamma=\Gamma_{a, b, V} \cap \Gamma_{-b, a, V^{*}}$. Moreover, if we let

$$
A_{0}=\frac{c_{V}^{p}(a, b)}{C} \int_{\mathfrak{F}} N\left(\left(i x t^{1 / n} / d_{V}^{1 / n}\right)^{\frac{1}{2} p}\right) d^{*} x,
$$

which is equal to 0 unless $c_{V}^{p}(a, b)=1$, in which case by Lemma 4.1 it is equal to $C^{-1} \operatorname{vol}(\mathfrak{F})$, and similarly if we let

$$
B_{0}=\frac{c_{V^{*}}^{p}(-b, a)}{C} \int_{\mathfrak{F}^{\prime}} N\left(\left(i x t^{1 / n} / d_{V}^{1 / n}\right)^{\frac{1}{2} p}\right) d^{*} x,
$$

then

$$
f(t)=A_{0}+O\left(e^{-\alpha t^{1 / n}}\right) \quad \text { and } \quad g(t)=B_{0}+O\left(e^{-\beta t^{1 / n}}\right)
$$

for $t \rightarrow \infty$ and suitable $\alpha, \beta>0$.

Proof. The proof of (4.8) follows from easy estimates. Let us prove (4.7). We have

$$
\begin{aligned}
f(1 / t) & =\frac{1}{C} \int_{\mathfrak{F}} \theta_{V}^{p}\left(a, b, \omega_{p}, i x t^{-1 / n} / d_{V}^{1 / n}\right) N\left(\left(i x t^{-1 / n} / d_{V}^{1 / n}\right)^{\frac{1}{2} p}\right) d^{*} x \\
= & \frac{1}{C} \int_{\mathfrak{F}} \theta_{V}^{p}\left(a, b, \omega_{p},-1 /\left(i x^{-1}\left(d_{V} t\right)^{1 / n}\right)\right) N\left(-1 /\left(i x^{-1}\left(d_{V} t\right)^{1 / n}\right)^{\frac{1}{2} p}\right) d^{*}\left(x^{-1}\right)
\end{aligned}
$$




$$
\begin{aligned}
& =\frac{1}{C} \int_{\mathfrak{F}^{-1}} \theta_{V}^{p}\left(a, b, \omega_{p},-1 /\left(i x\left(d_{V} t\right)^{1 / n}\right)\right) N\left(-1 /\left(i x\left(d_{V} t\right)^{1 / n}\right)^{\frac{1}{2} p}\right) d^{*} x \\
& =\frac{1}{d_{V} t C} \int_{\left(d_{V} t\right)^{1 / n} \mathfrak{F}^{-1}} \theta_{V}^{p}\left(a, b, \omega_{p},-1 /(i y)\right) N\left((-1 / i y)^{\frac{1}{2} p}\right) d^{*} y
\end{aligned}
$$

with $y=x\left(d_{V} t\right)^{1 / n}$. The second equality uses the fact that the transformation $x \mapsto x^{-1}$ fixes the Haar measure $d^{*} x$. The third equality uses the fact that $\frac{1}{d_{V} t} d^{*} y=d^{*} x$ where $d^{*} y$ is the corresponding measure on $\left(d_{V} t\right)^{1 / n} \mathfrak{F}^{-1}$ and $\mathfrak{F}^{-1}=\left\{x^{-1} \in \mathbf{S}: x \in \mathfrak{F}\right\}$. Now applying Theorem 3.1 to the last equality we find that

$$
\begin{aligned}
f(1 / t)= & \frac{\left(i^{\operatorname{Tr}(p)} e^{-2 \pi i\langle a, b\rangle} \sqrt{d_{V}}\right)^{-1}}{d_{V} t C} \\
& \times \int_{\left(d_{V} t\right)^{1 / n} \mathfrak{F}^{-1}} N\left(y^{p+\frac{1}{2} \cdot \mathbf{1}}\right) \theta_{V^{*}}^{p}\left(-b, a, \omega_{p}, i y\right) N\left((-1 / i y)^{\frac{1}{2} p}\right) d^{*} y \\
= & \frac{\left(i^{\operatorname{Tr}(p)} e^{-2 \pi i\langle a, b\rangle} \sqrt{d_{V}}\right)^{-1}}{d_{V} t C} \\
& \times \int_{\left(d_{V} t\right)^{1 / n} \mathfrak{F}^{-1}} N\left(y^{\frac{1}{2} \cdot \mathbf{1}}\right) \theta_{V^{*}}^{p}\left(-b, a, \omega_{p}, i y\right) N\left((i y)^{\frac{1}{2} p}\right) d^{*} y \\
= & (-i)^{\operatorname{Tr}(p)} \frac{\left(e^{-2 \pi i\langle a, b\rangle} \sqrt{d_{V} d_{V^{*}}}\right)^{-1}}{d_{V} d_{V^{*}} C} \sqrt{t} \\
& \times \int_{\left(d_{V} d_{V^{*}}\right)^{1 / n} \mathfrak{F}^{-1}} \theta_{V^{*}}^{p}\left(-b, a, \omega_{p}, i u t^{1 / n} / d_{V^{*}}^{1 / n}\right) N\left(\left(i u t^{1 / n} / d_{V^{*}}^{1 / n}\right)^{\frac{1}{2} p}\right) d^{*} u
\end{aligned}
$$

where $y=u t^{1 / n} / d_{V^{*}}^{1 / n}$ and $d y^{*}=\frac{t}{d_{V^{*}}} d^{*} u$ and where $d^{*} u$ is the corresponding measure on $\left(d_{V} / d_{V^{*}}\right)^{1 / n} \mathfrak{F}^{-1}$. A direct computation shows that $d_{V} d_{V^{*}}=1$ and that $\mathfrak{F}^{-1}$ is a fundamental domain for the action of $\iota\left(\Gamma_{a, b, V}\right)$ on $\mathbf{S}$. We can thus rewrite the last equality as

$$
\begin{aligned}
f(1 / t)= & \frac{(-i)^{\operatorname{Tr}(p)} e^{2 \pi i\langle a, b\rangle}}{C} \sqrt{t} \\
& \times \int_{\mathfrak{F}^{-1}} \theta_{V^{*}}^{p}\left(-b, a, \omega_{p}, i u t^{1 / n} / d_{V^{*}}^{1 / n}\right) N\left(\left(i u t^{1 / n} / d_{V^{*}}^{1 / n}\right)^{\frac{1}{2} p}\right) d^{*} u .
\end{aligned}
$$

Note that $\mathfrak{F}^{-1}$ is also a fundamental domain for the action of $\iota\left(\Gamma_{-b, a, V^{*}}\right)=$ $\iota\left(\Gamma_{a, b, V}\right)$ on $\mathbf{S}$. Therefore using (4.9) we deduce that

$$
\begin{aligned}
f(1 / t)= & \frac{\left(i^{\operatorname{Tr}(p)} e^{-2 \pi i\langle a, b\rangle}\right)^{-1}}{C} \sqrt{t} \\
& \times \int_{\mathfrak{F}^{\prime}} \theta_{V^{*}}^{p}\left(-b, a, \omega_{p}, i u t^{1 / n} / d_{V^{*}}^{1 / n}\right) N\left(\left(i u t^{1 / n} / d_{V^{*}}^{1 / n}\right)^{\frac{1}{2} p}\right) d^{*} u .
\end{aligned}
$$


We are now ready to prove Theorem 1.1.

Proof of Theorem 1.1. Assume that $\operatorname{Re}(s)>1$. From Proposition 4.1 we get

$$
\begin{aligned}
Z_{V}\left(a, b, \omega_{p}, 2 s\right) & =\int_{0}^{\infty}\left(f(t)-A_{0}\right) t^{s} \frac{d t}{t} \\
& =\int_{0}^{1}\left(f(t)-A_{0}\right) t^{s} \frac{d t}{t}+\int_{1}^{\infty}\left(f(t)-A_{0}\right) t^{s} \frac{d t}{t} \\
& =\int_{1}^{\infty} f(1 / t) t^{-s} \frac{d t}{t}+\int_{1}^{\infty}\left(f(t)-A_{0}\right) t^{s} \frac{d t}{t}-\frac{A_{0}}{s} .
\end{aligned}
$$

Substituting (4.7) into the first integral of the last equality we get

$$
\begin{aligned}
& Z_{V}\left(a, b, \omega_{p}, 2 s\right)=(-i)^{\operatorname{Tr}(p)} e^{2 \pi i\langle a, b\rangle} \int_{1}^{\infty}\left(g(t)-B_{0}\right) t^{-s+1 / 2} \frac{d t}{t} \\
& +\int_{1}^{\infty}\left(f(t)-A_{0}\right) t^{s} \frac{d t}{t}-\frac{A_{0}}{s}-\frac{B_{0}}{-s+1 / 2}(-i)^{\operatorname{Tr}(p)} e^{2 \pi i\langle a, b\rangle}
\end{aligned}
$$

The two integrals of the last equality converge for all complex number $s \in \mathbb{C}$. It follows from this that $Z_{V}\left(a, b, \omega_{p}, 2 s\right)$ admits an analytic continuation to all $s \in \mathbb{C} \backslash\{0,1 / 2\}$. This proves the first part of Theorem 1.1. The equality (4.10) can be rewritten as

$$
\begin{aligned}
i^{\operatorname{Tr}(p)} e^{-2 \pi i\langle a, b\rangle} Z_{V}\left(a, b, \omega_{p}, 2 s\right) & \\
= & \int_{1}^{\infty}\left(g(t)-B_{0}\right) t^{-s+1 / 2} \frac{d t}{t}+e^{-2 \pi i\langle a, b\rangle} \int_{1}^{\infty}\left(f(t)-A_{0}\right) t^{s} \frac{d t}{t} \\
& \quad-\frac{B_{0}}{-s+1 / 2}-\frac{A_{0}}{s} i^{\operatorname{Tr}(p)} e^{-2 \pi i\langle a, b\rangle} .
\end{aligned}
$$

In the exact same way as we obtained (4.10) one has that

$$
\begin{aligned}
& Z_{V^{*}}\left(-b, a, \omega_{p}, 2 s\right)=(-i)^{\operatorname{Tr}(p)} e^{-2 \pi i\langle a, b\rangle} \int_{1}^{\infty}\left(f(t)-A_{0}\right) t^{-s+1 / 2} \frac{d t}{t} \\
& +\int_{1}^{\infty}\left(g(t)-B_{0}\right) t^{s} \frac{d t}{t}-\frac{A_{0}}{-s+1 / 2}(-i)^{\operatorname{Tr}(p)} e^{-2 \pi i\langle a, b\rangle}-\frac{B_{0}}{s} .
\end{aligned}
$$

Replacing $s$ by $-s+1 / 2$ in (4.11) and comparing the result with (4.12) reveals that

$$
(-i)^{\operatorname{Tr}(p)} e^{-2 \pi i\langle a, b\rangle} Z_{V}\left(a, b, \omega_{p}, 2(-s+1 / 2)\right)=Z_{V^{*}}\left(-b, a, \omega_{p}, 2 s\right) .
$$

This concludes the proof. 
5. Partial zeta functions twisted by sign characters. Let $K$ be a number field of degree $n=r_{1}+2 r_{2}$ over $\mathbb{Q}$ with different ideal $\mathfrak{d}_{K}$. Let $\mathfrak{f}$ be an integral ideal of $\mathcal{O}_{K}$. Choose an integral ideal $\mathfrak{b}$ which is prime to $\mathfrak{f}$ and consider the lattice $V=\mathfrak{b} / \mathfrak{f d}_{K}$. Note that $V^{*}=\mathfrak{f b}^{-1}$. Let $a=0, b=-1$ and $p=\left(p_{\tau}\right)_{\tau}$ be a strictly admissible tuple corresponding to a sign character $\omega_{p}:\left(K \otimes_{\mathbb{Q}} \mathbb{R}\right)^{\times} \rightarrow\{ \pm 1\}$. By definition we have

$$
\Gamma_{0,-1, V}=\mathcal{O}_{K}(\infty)^{\times} \cap\left(1+\mathfrak{f b}^{-1}\right),
$$

where $\mathcal{O}_{K}(\infty)^{\times}$corresponds to the group of totally positive units of $\mathcal{O}_{K}$. A direct computation shows that

$$
\Psi_{V}\left(0,-1, \omega_{p}, s\right)=\mathbf{N}_{K / \mathbb{Q}}\left(\frac{\mathfrak{b}}{\mathfrak{d}_{K} \mathfrak{f}}\right)^{s} \sum_{\left\{0 \neq \mu \in \mathfrak{b} / \mathfrak{f} \mathfrak{d}_{K}\right\} / \Gamma} \omega_{p}(\mu) \frac{e^{2 \pi i \operatorname{Tr}_{K / \mathbb{Q}}(\mu)}}{\left|\mathbf{N}_{K / \mathbb{Q}}(\mu)\right|^{s}}
$$

for $\operatorname{Re}(s)>1$, where $\Gamma=\Gamma_{0,-1, V}$. When $\omega_{p}$ is trivial, the right hand side of the last equality is an example of what we call a zeta function twisted by an additive character. Similarly one has

$$
\begin{aligned}
\Psi_{V^{*}}\left(1,0, \omega_{p}, s\right) & =\mathbf{N}_{K / \mathbb{Q}}\left(\mathfrak{f} \mathfrak{b}^{-1}\right)^{s} \sum_{\left\{0 \neq \mu \in 1+\mathfrak{f b}^{-1}\right\} / \Gamma} \frac{\omega_{p}(\mu)}{|\mathbf{N}(\mu)|^{s}} \\
& =\mathbf{N}_{K / \mathbb{Q}}(\mathfrak{f})^{s}\left(\sum_{\left\{0 \neq \mu \in 1+\mathfrak{f} \mathfrak{b}^{-1}\right\} / \Gamma} \frac{\omega_{p}(\mu)}{\left|\mathbf{N}_{K / \mathbb{Q}}(\mu \mathfrak{b})\right|^{s}}\right)
\end{aligned}
$$

for $\operatorname{Re}(s)>1$. The right hand side of the last equality is an example of what we call a partial zeta function twisted by a sign character. From Theorem 1.1 we deduce that

$$
(-i)^{\operatorname{Tr}(p)} \frac{F_{V}^{p}(s / 2)}{F_{V^{*}}^{p}((1-s) / 2)} \Psi_{V}\left(0,-1, \omega_{p}, s\right)=\Psi_{V^{*}}\left(1,0, \omega_{p}, 1-s\right),
$$

where $F_{V}^{p}(s / 2) / F_{V^{*}}^{p}((1-s) / 2)$ is given explicitly by

$$
\begin{aligned}
& \frac{F_{V}^{p}(s / 2)}{F_{V^{*}}^{p}((1-s) / 2)} \\
& =\frac{\left|d_{K}\right|^{s / 2} \pi^{-n s / 2}}{\left|d_{K}\right|^{(1-s) / 2} \pi^{-n(1-s) / 2}} \frac{\prod_{i=1}^{r_{1}} \Gamma\left(\left(s+p_{i}\right) / 2\right)\left(2^{1-s} \Gamma(s)\right)^{r_{2}}}{\prod_{i=1}^{r_{1}} \Gamma\left(\left(1-s+p_{i}\right) / 2\right)\left(2^{s} \Gamma(1-s)\right)^{r_{2}}} .
\end{aligned}
$$

For any $s \in \mathbb{Z}_{\geq 1}$ the function $\Psi_{V}\left(0,-1, \omega_{p}, s\right)$ is holomorphic at $s$ (for $s=1$ this uses the fact that $\mathfrak{f} \nmid \mathfrak{b}$ ). From (5.2) and (5.3) we deduce that for $s \in \mathbb{Z}_{\geq 1}$ the value $\Psi_{V^{*}}\left(1,0, \omega_{p}, 1-s\right)$ is not zero only when

(1) $r_{2}=0, s \equiv 0(\bmod 2)$ and $p_{i}=0$ for all $i$

or

(2) $r_{2}=0, s \equiv 1(\bmod 2)$ and $p_{i}=1$ for all $i$. 
For a given totally real number field $K$, the relationships between special values at negative integers of $\Psi_{V^{*}}\left(1,0, \omega_{p}, s\right)$ and classical partial zeta functions as in (1.8) were treated in [Cha07b].

REMark 5.1. In [Del79], Deligne introduced the notion of critical integers for an $L$-function $L(M, s)$ attached to a motive $M$. In the absence of a motive associated to the zeta function $\Psi_{V}(a, b, \omega, s)$, it seems to be still useful to introduce the notion of critical integer. We will say that an integer $n$ is critical for the zeta function $\Psi_{V}\left(a, b, \omega_{p}, s\right)$ if

$$
F_{V}^{p}(n / 2) \neq \infty \quad \text { and } \quad F_{V^{*}}^{p}((1-n) / 2) \neq \infty \Leftrightarrow \frac{F_{V}^{p}(n / 2)}{F_{V^{*}}^{p}((1-n) / 2)} \neq 0, \infty .
$$

Let $S$ be the set of critical integers of $\Psi_{V}\left(a, b, \omega_{p}, s\right)$. A direct calculation, similar to the one we did previously, shows that

$$
S= \begin{cases}2 \mathbb{Z}_{\geq 1} \cup\left(1+2 \mathbb{Z}_{\leq-1}\right) & \text { if } r_{2}=0, \omega_{p}=1, \\ \left(1+2 \mathbb{Z}_{\geq 0}\right) \cup 2 \mathbb{Z}_{\leq 0} & \text { if } r_{2}=0, \omega_{p}=\operatorname{sign} \circ \mathbf{N}_{K / \mathbb{Q}}, \\ \emptyset & \text { otherwise. }\end{cases}
$$

\section{References}

[CN79] P. Cassou-Noguès, Valeurs aux entiers négatifs des fonctions zêta p-adiques, Invent. Math. 51 (1979), 29-59.

[Cha07a] H. Chapdelaine, p-units and Gauss sums, submitted.

[Cha07b] -, On some arithmetic properties of partial zeta functions weighted by sign characters, submitted.

[Del79] P. Deligne, Valeurs de fonctions $L$ et périodes d'intégrales, in: Proc. Sympos. Pure Math. 33, Part 2, Amer. Math. Soc., Providence, RI, 1979, 313-346.

[DR80] P. Deligne and K. A. Ribet, Values of abelian L-functions at negative integers over totally real fields, Invent. Math. 59 (1980), 227-286.

[Hec17] E. Hecke, Über die Zetafunktion beliebiger algebraischer Zahlkörper, Nachr. Königl. Ges. Wiss. Göttingen Math.-Phys. Kl. 1917, 77-89; Mathematische Werke, Vandenhoeck \& Ruprecht, Göttingen, 1959, 159-171.

[Hec20] - Eine neue Art von Zetafunktionen und ihre Beziehungen zur Verteilung der Primzahlen, zweite Mitteilung, Math. Z. 6 (1920), 11-51; Mathematische Werke, Vandenhoeck \& Ruprecht, Göttingen, 1959, 249-289.

[Kli62] H. Klingen, Über die Werte der Dedekindschen Zetafunktion, Math. Ann. 145 (1962), 265-272.

[Neu99] J. Neukirch, Algebraic Number Theory, Springer, Grundlehren Math. Wiss. 322, Berlin, 1999.

[Rie59] B. Riemann, Über die Anzahl der Primzahlen unter einer gegebenen Grösse, Monatsber. Berliner Akad. 1859; Gesammelte Mathematische Werke, Springer, Berlin, 1990, 177-187.

[Shi76] T. Shintani, On evaluation of zeta functions of totally real algebraic number fields at non-positive integers, J. Fac. Sci. Univ. Tokyo Sect. I A 23 (1976), 393-417. 
[Sie69] C. L. Siegel, Berechnung von Zetafunktionen an ganzzahligen Stellen, Nachr. Akad. Wiss. Göttingen Math.-Phys. Kl. II, 1969, 87-102.

[Sie70] C. L. Siegel, Über die Fourierschen Koeffizienten von Modulformen, ibid. 1970, $15-56$.

Département de mathématiques et de statistique

Université Laval

Québec, QC, Canada G1K 7P4

E-mail: hugo.chapdelaine@mat.ulaval.ca

http://www.mat.ulaval.ca/le_departement/pages_personnelles/hugo_chapdelaine

Received on 10.3.2008

and in revised form on 26.8.2008 